\title{
Inversion simultanée des données sismiques et des données de production
}

\author{
L. Pianelo' , D. Guérillot ${ }^{1}$ et T. Gallouët ${ }^{2}$ \\ 1 Institut français du pétrole, 1 et 4, avenue de Bois-Préau, 92852 Rueil-Malmaison Cedex - France \\ 2 Université d'Aix-Marseille I, CMI, 13453 Marseille Cedex 13 - France \\ e-mail : laurent.pianelo@ifp.fr - thierry.gallouet@cmi.univ-mrs.fr
}

\begin{abstract}
Résumé - On utilise, pour l'interprétation des mesures de production aux puits, ainsi que pour l'interprétation des profils sismiques, des procédures d'inversion. Elles permettent d'essayer, à partir d'un modèle géologique maillé, de caler des simulations numériques sur des mesures de terrain, ceci en faisant varier les paramètres de ce modèle. Malheureusement, un manque de données permet souvent à plusieurs modèles de résoudre le problème inverse. La gestion des incertitudes devient alors complexe. Une nouvelle méthode est donc proposée pour inverser simultanément les deux modèles (sismique et gisement) afin de réduire le nombre de scénarios possibles. On se sert ici des relations de laboratoire pour relier la perméabilité absolue du milieu à son impédance acoustique. On ajoute alors de l'information aux deux problèmes inverses et on améliore ainsi considérablement les modèles. In fine, on cherche à fournir à l'ingénierie de gisement les moyens de prévoir le comportement des gisements pétroliers hétérogènes et d'évaluer l'incertitude inhérente à ces prévisions.

Sont présentés dans cet article les problèmes et procédures mathématiques employées comme modèles directs et inverses, ainsi que des tests numériques validant la méthode.

Mots-clés : caractérisation des réservoirs, interprétation sismique, essais de puits, historique de production, inversion, état adjoint, gradient, couplage d'inversion.
\end{abstract}

\footnotetext{
Abstract - Coupled Matching of the Production Data and the Seismic Data - Matching procedures are often used in reservoir production to improve geological models. In reservoir engineering, history matching leads to update petrophysical parameters in fluid flow simulators to fit the results of the calculations with observed data. In the same line, seismic parameters are inverted to allow the numerical recovery of seismic acquisitions. However, it is well known that these inverse problems are poorly constrained.

The idea of this original work is to match simultaneously both the permeability and acoustic impedance of the reservoir, for an enhancement of the resulting geological model. To do so, both parameters are linked using either observed relations and/or the classic Wyllie (porosity-impedance) and CarmanKozeny (porosity-permeability) relationships. Hence, production data are added to the seismic match, and seismic observations are used for the permeability recovery.

The work consists in developing numerical prototypes of a $1 D$ fluid flow simulator and a $1 D$ seismic acquisition simulator. Then, in implementing the coupled inversion loop of the permeability and acoustic impedance of the two models, we can test our theory on a $1 D$ case.

Comparison of the coupled matching with the two classical ones demonstrates the efficiency of our method. We reduce significantly the number of possible solutions, and then the number of scenarios. In addition, the augmentation of information leads to a natural improvement of the obtained models,
} 
especially in the spatial localization of the permeability contrasts. The improvement is significant, at the same time in the distribution of the two inverted parameters, and in the rapidity of the operation.

This work is an important step in a way of data integration. It allows to use the available hard and soft data, as the knowledge of the depositional environment (like geostatistical models), seismic information (like surface seismograms), and/or dynamic information (like well-test and production data), and leads to a better reservoir characterization. This original algorithm could also be useful in reservoir monitoring, history matching and in optimization of production.

Keywords: history matching, seismic interpretation, reservoir characterization, matching procedures, inversion, adjoint state, gradient methods, coupled matching.

\section{EXPOSÉ DU SUJET}

Une des grandes voies de recherche actuellement dans le milieu pétrolier est d'arriver à donner de meilleures descriptions de l'architecture interne des réservoirs. Pour cela, les ingénieurs ont essentiellement à leur disposition trois types de données :

- les modèles élaborés par les études géologiques ;

- les données dynamiques (gisement);

- les données statiques (sismiques).

Des modèles différents et divers sont créés, suivant le type de données employées.

Le but de cette thèse est d'utiliser l'ensemble des données (sismiques et de gisement) pour ainsi améliorer sensiblement les modèles géologiques.

Pour construire de bons modèles de réservoirs, deux techniques similaires, mais basées sur des données différentes, sont couramment utilisées :

- inversion des informations statiques : ces informations proviennent des traitements et interprétations sismiques. Les données géophysiques qui en résultent permettent d'établir des cartes du réservoir (sismique 2D). Le paramètre géophysique le plus souvent utilisé pour l'inversion est l'impédance acoustique du milieu ;

- inversion des informations dynamiques : dynamiques, puisqu'elles peuvent s'étendre sur quelques jours pour les essais de puits (et même plusieurs années pour les données de production). Mais ces informations sont ponctuelles, car elles sont localisées en des endroits précis : les puits, ou plutôt le long des puits. Les renseignements pétrophysiques obtenus seront axés sur la verticale du réservoir. Les paramètres d'inversion vont être, dans ce cas, la perméabilité totale du milieu et sa porosité.

L'inversion est un algorithme d'optimisation. Celui-ci modifie de proche en proche un modèle géologique donné $a$ priori en fonction de l'étude des caractéristiques observables aux puits. Ainsi, on rapproche au mieux les réponses (dynamiques ou statiques) du modèle du réservoir et du réservoir observé, à travers la minimisation d'une fonction coût $J$.

Dans les deux cas, l'algorithme d'inversion est le même (fig. 1).

Une étude en gisement, tout comme une interprétation sismique, peut durer plusieurs mois. Malheureusement, ces travaux sont généralement faits par des équipes différentes. De plus, chaque direction de travail aboutit à un nombre trop important de solutions possibles.

La direction principale de recherche de cette thèse est d'inverser les deux paramètres (dynamique et statique) simultanément.

Il faut pour cela trouver tout d'abord un modèle géologique donné a priori qui puisse être relié de façon cohérente aux deux paramètres, puis, à partir de ce modèle, considérer non plus un, mais deux problèmes directs :

- le problème d'écoulement ;

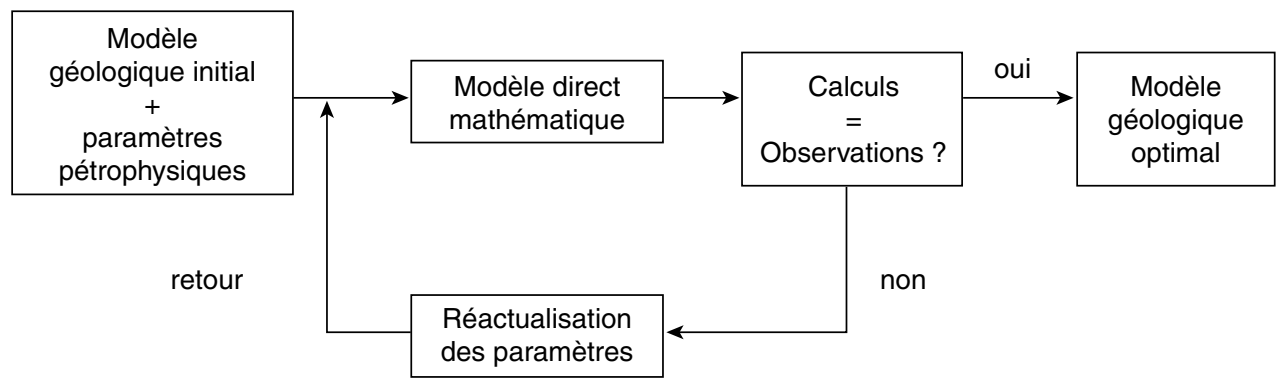

Figure 1

Algorithme d'inversion.

Inversion loop. 
- le problème géophysique modélisé par l'équation des ondes,

en ajoutant par exemple l'information sismique comme contrainte « régionale ». La liaison entre les problèmes d'inversion se fait ici en utilisant des relations (algébriques) de laboratoire.

Les modèles obtenus vont donc satisfaire à la fois les contraintes dynamiques et statiques. Ce qui amène tout naturellement à :

- une diminution considérable du nombre de solutions possibles ;

- une amélioration sensible des modèles en matière de caractérisation des réservoirs.

\section{LE PROBLÈME INVERSE}

\subsection{Théorie}

Un problème inverse consiste à déterminer une quantité physique $p$, qui est difficilement mesurable, en analysant des quantités mesurables $d$ (Tarantola, 1987). Un modèle mathématique fournit une relation entre $d$ et $p$, que nous noterons $d=\phi(p)$. Ce que nous appelons problème direct consiste à calculer $d$ lorsque $p$ est (supposé) connu. Ce calcul nécessite en général de résoudre une ou plusieurs équation(s) aux dérivées partielles. Le problème inverse consiste à essayer d'identifier $p$ d'après $d$. En pratique, nous disposons de mesures expérimentales $\phi$ et nous cherchons le (ou un ?) paramètre $\hat{p}$ tel que $\hat{d}=\phi(\hat{p})$. Une méthode classique de résolution d'un tel problème est la méthode des moindres carrés (Bourbie et al., 1986 ; Guérillot et al., 1998). Nous cherchons $\hat{p}$ tel que :

$$
J(\hat{p})=\min _{p} J(p)
$$

où $J(p)=\|\hat{d}-\phi(p)\|^{2}$ est la fonction objectif.

Nous considérons ici la classe des problèmes inverses pour lesquels trouver $\phi$ consiste à résoudre une ou plusieurs équation(s) aux dérivées partielles. Dans ce cadre, la fonction objectif est coûteuse en temps de calcul. Nous nous plaçons également dans le cas où la taille du vecteur de paramètres $p$ est telle qu'une optimisation sans calcul de gradient est difficilement envisageable.

Le code d'optimisation de $J$ réclame alors l'écriture de deux procédures qui dépendent du problème : l'une résout le problème direct et calcule la fonction objectif $J$, et l'autre calcule le gradient de $J$. La procédure qui calcule $\nabla J$ peut être obtenue de deux manières :

- $\nabla J$ pourrait être calculé en faisant légèrement évoluer les paramètres un par un et en utilisant une formule de type différence-finies. Cette approche est très coûteuse en temps de calcul, surtout lorsque $p$ est de grande taille.
Elle donne seulement une approximation du gradient et ne permet donc pas une optimisation très précise de la fonction objectif $J$. De plus, il est très difficile de choisir le pas dans la formule de différence-finies ;

- pour ces raisons, nous optons plutôt pour un calcul exact du gradient. Cette approche est la plus efficace, que ce soit en temps de calcul (pour un grand nombre de paramètres) ou pour l'optimisation (le gradient est exact). En revanche, elle est fastidieuse, difficile à mettre au point et source de nombreuses erreurs. De plus, une fois la méthode mise en œuvre pour un modèle, si une modification doit être apportée au schéma numérique, tout est à recommencer.

\subsection{Méthode de l'état adjoint}

Nous allons expliquer ici brièvement les fondements de la méthode de l'état adjoint dont nous nous sommes servis pour l'inversion des paramètres des deux équations étudiées dans le cadre de ce travail : l'équation d'écoulement et l'équation des ondes (Bamberger et al., 1977 ; Jégou, 1997 ; Edoa, 1999).

Comme nous l'avons déjà signalé, la fonction $\phi$ représente ici la résolution d'une ou de plusieurs équation(s) aux dérivées partielles. La méthode de l'état adjoint consiste à construire un lagrangien $L(u, v, p)$, où $u$ est la variable d'état, $v$ la variable adjointe et $p$ le paramètre à estimer. Ce lagrangien est formé de la fonction objectif $J$ (considérée ici comme une fonction de la variable d'état) et de la formulation variationnelle du problème direct multipliée par un multiplicateur de Lagrange $v$. Ainsi, nous pouvons écrire formellement :

$$
\mathrm{d} J=\frac{\partial L}{\partial p}(u(p), v, p) \mathrm{d} p
$$

où $u(p)$ est la solution des équations d'état avec la valeur courante $p$ du paramètre, et $v$ la solution de l'équation adjointe suivante :

$$
\frac{\partial L}{\partial u}(u(p), v, p) \mathrm{d} u=0
$$

Pour résoudre le problème inverse, nous pourrions appliquer cette méthode aux équations continues et construire une approximation des équations adjointes continues ainsi obtenues.

En pratique, une bien meilleure méthode consiste à construire une approximation des équations d'état et de la fonction objectif $J$. Il faut ensuite définir un lagrangien discret et des équations adjointes discrètes, et calculer un gradient discret. Le gradient obtenu est exact pour le problème approché qui est effectivement résolu. L'optimisation n'en est que plus efficace. 
Maintenant que nous avons calculé un gradient exact de la fonction coût, nous déterminons $\alpha \geq 0$ minimisant :

$$
g(\alpha)=J\left(p^{0}-\alpha \nabla J\right)
$$

où : $p^{0}=\left(p_{1}^{0}, p_{2}^{0}, \ldots, p_{n x}^{0}\right)^{T}$ est le dernier point obtenu,

et :

$$
-\nabla J=-\left(\frac{\partial J}{\partial p_{1}}, \frac{\partial J}{\partial p_{2}}, \cdots \frac{\partial J}{\partial p_{n x}}\right)^{T}
$$

est la direction de déplacement (ou direction de descente).

Nous avons choisi d'appliquer la règle de Goldstein (1967), qui assure la convergence de l'algorithme en un nombre très limité d'itérations (Minoux, 1983).

Il nous suffit après cela de réactualiser le paramètre $p$, en passant de l'itération $n$ à l'itération $n+1$ par le calcul :

$$
p^{n+1}=p^{n}-\alpha \nabla J
$$

\section{LES MODÈLES PHYSIQUES ET MATHÉMATIQUES}

\subsection{Le modèle gisement}

Il s'agit ici d'exposer les diverses techniques utilisées et les différents modèles associés pour inverser le paramètre perméabilités absolues de l'équation d'écoulement des fluides. Pour cela, il a été choisi l'exemple simple unidimensionnel d'un fluide s'écoulant dans un réservoir.

Le schéma numérique utilisé pour résoudre le modèle direct est un schéma en différence-finies. Une description de la perméabilité aux interfaces inspirée des schémas en volume-finis permet de prendre en compte la continuité du flux aux interfaces (Gallouët, 1992; Baranger, 1977).

Considérons un bassin sédimentaire. À l'intérieur de ce bassin se trouve un réservoir dans lequel deux puits, un injecteur en entrée, un producteur à la sortie, vont faire s'écouler un fluide (fig. 2).

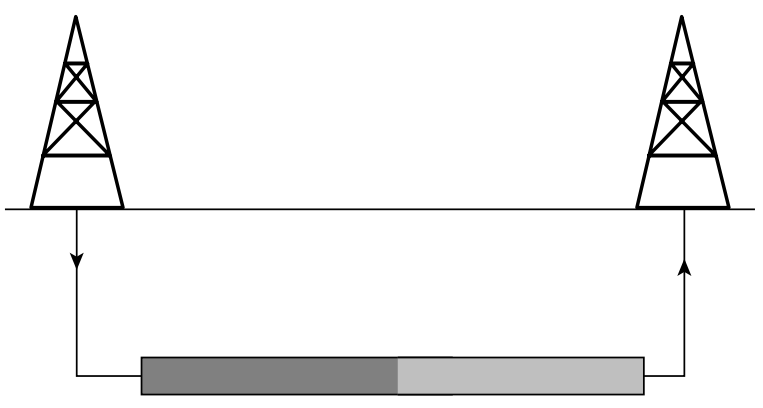

Figure 2

Injection de fluide dans un réservoir entre deux puits.

Fluid flow between two wells.
On peut schématiser le réservoir comme une barre cylindrique de longueur $L$, dont les deux extrémités sont en contact avec des « sources » de débit. Connaissant la pression du fluide Po à l'instant initial, son évolution sur l'intervalle de temps $[0, T]$ est régie par les équations suivantes (Rahon et al., 1996 ; Guérillot et al., 1998):

$$
\begin{cases}p(x, 0)=p_{0}(x) & \forall x \in[0, L] \\ -k(0) \frac{\partial p}{\partial x}(0, t)=q_{0}(t) & \forall t \in] 0, T] \\ \frac{\partial p}{\partial t}+\frac{\partial}{\partial x}\left(-k(x) \frac{\partial p}{\partial x}\right)=0 & \forall x \in[0, L] \text { et } \forall t \in] 0, T] \\ p(L, t)=p_{L} & \forall t \in] 0, T]\end{cases}
$$

où :

$p(x, t)$ pression du fluide à l'instant $t$ et à la position $x$

$k(x)$ perméabilité de la roche à la position $x$

$q_{0}(t)$ débit injecté à l'instant $t$

$p_{L} \quad$ pression à l'extrémité de la barre $(x=L)$

Supposons que le réservoir soit un «assemblage de tranches » (fig. 3) de lithologies homogènes, de perméabilités différentes qu'il s'agit d'identifier.

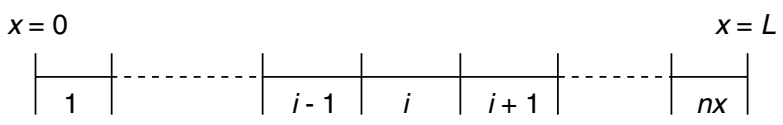

Figure 3

Réservoir maillé.

Meshed reservoir.

Pour ce faire, nous disposons de mesures de pression $\hat{p}$ au puits injecteur, en $x=0$.

Nous pouvons donc construire la fonction objectif suivante :

$$
J(k)=\frac{1}{2} \int_{0}^{T}(p(0, t)-\hat{p}(t))^{2} \mathrm{~d} t
$$

(pas de terme de régularisation ici, ni d'informations $a$ priori).

Et on procède ainsi à l'inversion du paramètre $k$ par des méthodes d'optimisation classiques, comme la méthode de l'état adjoint.

\subsection{Le problème sismique}

L'équation des ondes est une équation hyperbolique, d'inconnue $y$ qui désigne l'ébranlement du sous-sol ( $y$ dépend du temps et de la profondeur), et de paramètre $\sigma$ qui désigne l'impédance acoustique du milieu et dépend de la profondeur. Pour traiter cette équation, on emploie le même type de 
schéma que pour l'équation d'écoulement, c'est-à-dire un schéma en différence-finies, où nous utilisons une description des impédances aux interfaces inspirée des schémas en volume-finis. La différence est que nous obtenons ici, en raison des conditions initiales, un schéma explicite (Céa, 1971 ; Baranger, 1977).

Considérons un bassin sédimentaire. Celui-ci est composé de plusieurs couches géologiques, chaque couche ayant une impédance acoustique propre.

Les méthodes d'exploration sismique consistent à provoquer des ébranlements dans le sous-sol et à observer en surface les ondes réfléchies sur les couches géologiques ou réfractées le long de certaines interfaces.

Les ondes réfléchies remontent vers la surface où elles sont enregistrées : c'est le principe de la sismique-réflexion (fig. 4).

Les interfaces géologiques repérées en sismique-réflexion sont les surfaces de séparation des milieux d'impédances acoustiques différentes. L'impédance acoustique est par définition le produit de la masse volumique de la roche par la vitesse de propagation de l'onde sismique (Boyer et Mari, 1994 ; Bourbie et al., 1986).

Le milieu étant au repos à l'instant initial $t=0$, et connaissant l'impulsion en pression $g(t)$ communiquée au milieu en surface à chaque instant $t$, l'ébranlement $y(z, t)$ est la solution du système suivant ( $z$ désigne le temps de parcours de l'ébranlement et $\sigma(z)$ l'impédance acoustique dépendant du temps de parcours - une impédance différente par couche géologique) (Bamberger et al., 1977):

$$
\begin{cases}\sigma(z) \frac{\partial^{2} y}{\partial t^{2}}-\frac{\partial}{\partial z}\left(\sigma(z) \frac{\partial y}{\partial z}\right)=0 & \text { pour } z>0 \text { et pour } t>0 \\ y(z, 0)=\frac{\partial y}{\partial t}(z, 0)=0 & \text { pour } z \geq 0 \\ -\sigma(0) \frac{\partial y}{\partial z}(0, t)=g(t) & \text { pour } t>0 \\ y(L, t)=0 & \text { pour } t>0\end{cases}
$$

Si nous procédons au même découpage du sous-sol que lors du chapitre précédent, mais en vertical cette fois-ci, nous avons donc autant d'impédances acoustiques qu'il y a de couches, des impédances qu'il s'agit d'identifier.

Ici aussi, nous avons des mesures, qui sont les enregistrements de l'ébranlement en surface (en $z=0$ ), notées $\hat{y}$ et qui dépendent du temps.

Nous pouvons donc construire la fonction coût ainsi (écriture la plus simple, sans tenir compte d'informations $a$ priori):

$$
J(\sigma)=\frac{1}{2} \int_{0}^{T}(y(0, t)-\hat{y}(t))^{2} \mathrm{~d} t
$$

Elle nous permettra de réaliser l'inversion de l'impédance acoustique.

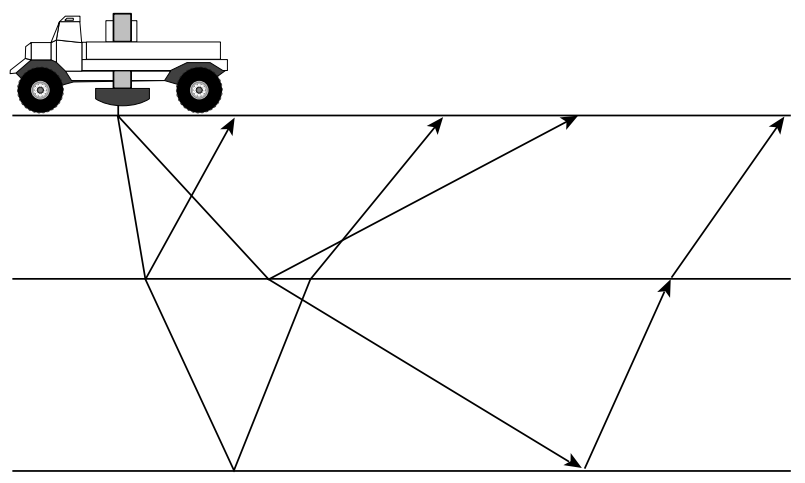

Figure 4

Principe de la sismique-réflexion.

Reflection seismic data acquisition.

\section{PROBLÈME : MANQUE DE DONNÉES}

\subsection{Explications}

Comme on peut le constater, la réussite de l'inversion dépend grandement de la qualité et de la quantité des observations dont on dispose. En effet, plus celles-ci seront nombreuses, meilleure sera la qualité du résultat de l'inversion. Un manque de données entraîne une information moyennée sur le paramètre que l'on cherche à inverser.

Autrement dit, un problème inverse réalisé sur deux mailles informées en paramètres $p_{1}$ et $p_{2}$ avec un nombre de données insuffisant va nous permettre de connaître seulement la moyenne des deux paramètres. En gisement par exemple, c'est la moyenne harmonique $p_{0}$ (fig. 5) des deux paramètres qui peut nous être retournée comme résultat.

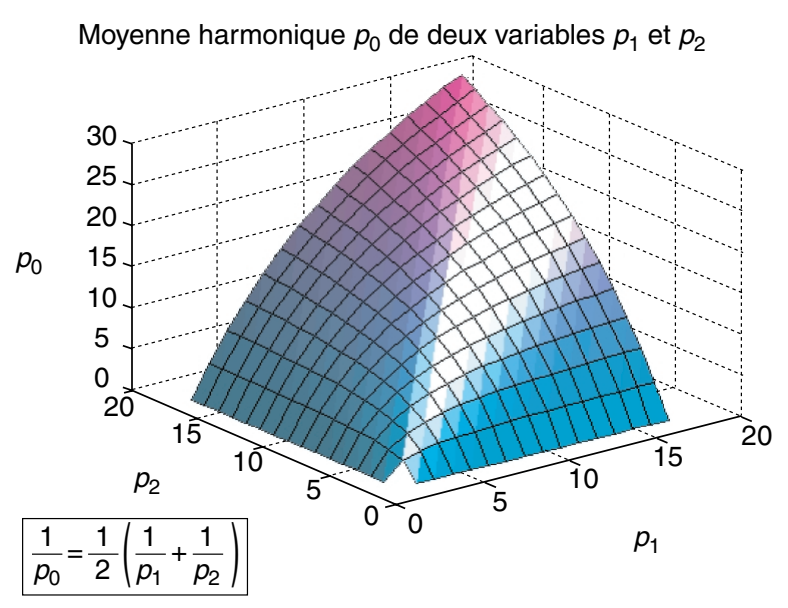

Figure 5

Moyenne harmonique.

Harmonic average. 

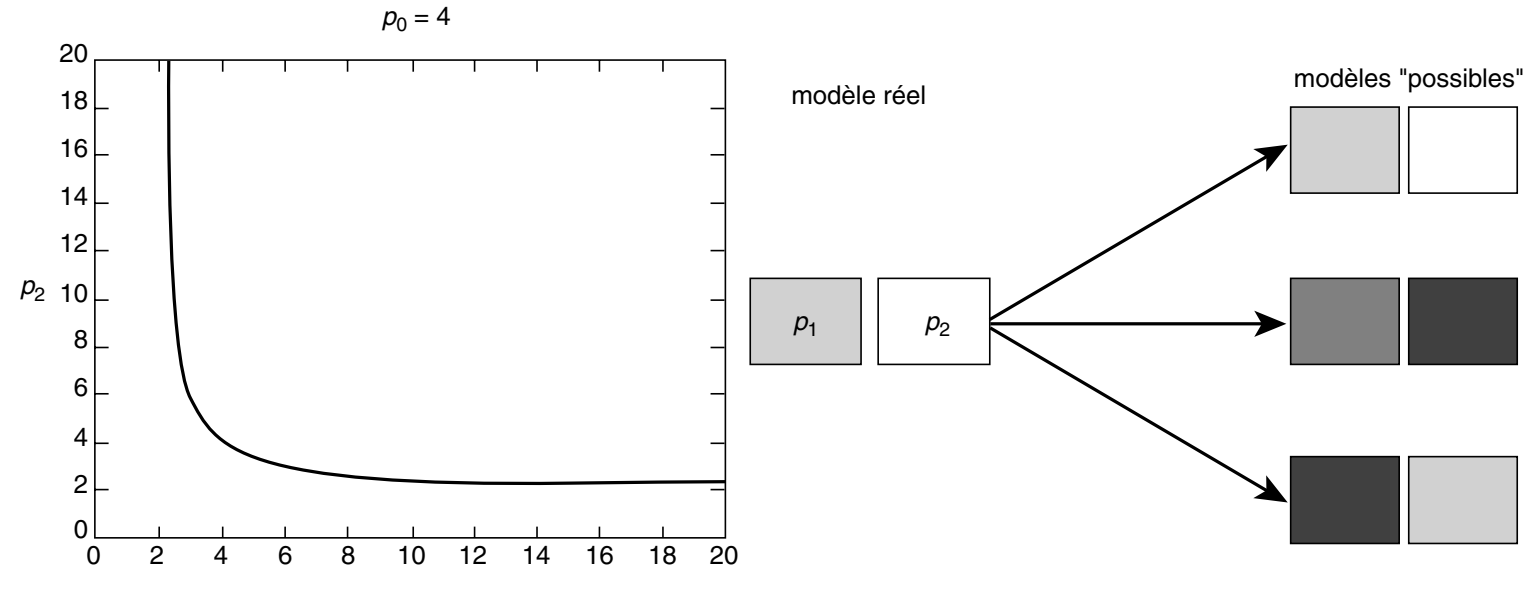

$p_{1}$

Figure 6

Pluralité des paramètres $p_{1}$ et $p_{2}$ de moyenne harmonique $p_{0}$.

Several sets of parameters $p_{1}$ and $p_{2}$ having harmonic mean $p_{0}$.

Le problème est que l'on dispose alors d'un grand nombre de choix possibles comme paramètres $p_{1}$ et $p_{2}$ de moyenne harmonique $p_{0}$ (fig. 6).

Cela a pour conséquence que l'on dispose de plusieurs modèles sur lesquels les simulations (gisement ou sismique) vont caler aux observations. Comment choisir alors le «meilleur » modèle, autrement dit le plus proche de la réalité ?

\subsection{Illustration numérique}

Une simulation 1D d'écoulement a été réalisée dans un réservoir. Celui-ci était composé d'une vingtaine de mailles, avec une perméabilité $k_{i}$ par maille (au centre de la maille). Il s'agissait d'un milieu trimodal, représentant un milieu perméable (par exemple du grès) coupé par une barrière imperméable (par exemple de l'argile) (fig. 7). Le but était de

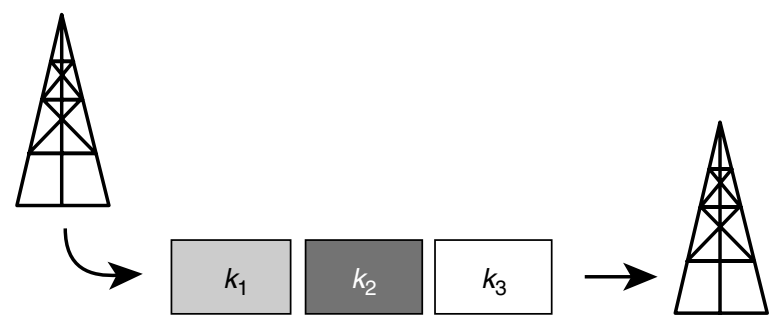

Figure 7

Simulation d'écoulement.

Fluid flow modeling. savoir si l'inversion du modèle permettait de retrouver le gradient de perméabilité. Les données étaient les pressions enregistrées au puits injecteur au cours du temps.

Les résultats obtenus par l'inversion sont donnés en figures $8 \mathrm{a}$ et $8 \mathrm{~b}$ (gradient calculé par la méthode de l'état adjoint).

Comme on peut le voir sur ces résultats, la répartition des perméabilités obtenues après l'inversion est très éloignée de la répartition « réelle » (ou plutôt de celle qui a servi à créer les données du puits injecteur). Pourtant, les réponses du modèle direct appliqué à cette répartition calent parfaitement aux données (fig. $8 b$ ). Cela prouve que plusieurs modèles, parfois très éloignés du modèle réel, peuvent résoudre le problème inverse.

\section{SOLUTION : COUPLAGE DES INVERSIONS}

\subsection{Explications}

L'idée de la thèse est donc d'inverser simultanément le paramètre gisement (perméabilité absolue) et le paramètre sismique (impédance acoustique). En reliant ces deux paramètres, on ajoute ainsi de l'information à chaque inversion, et on réduit l'intervalle de valeurs « admissibles » de chacun des paramètres (fig. 9).

L'apport d'information doit non seulement réduire le nombre de solutions possibles (car une contrainte est ajoutée), mais aussi améliorer sensiblement les modèles obtenus par rapport au modèle réel.

Voyons maintenant quelles relations on utilise entre la perméabilité absolue et l'impédance du milieu. 


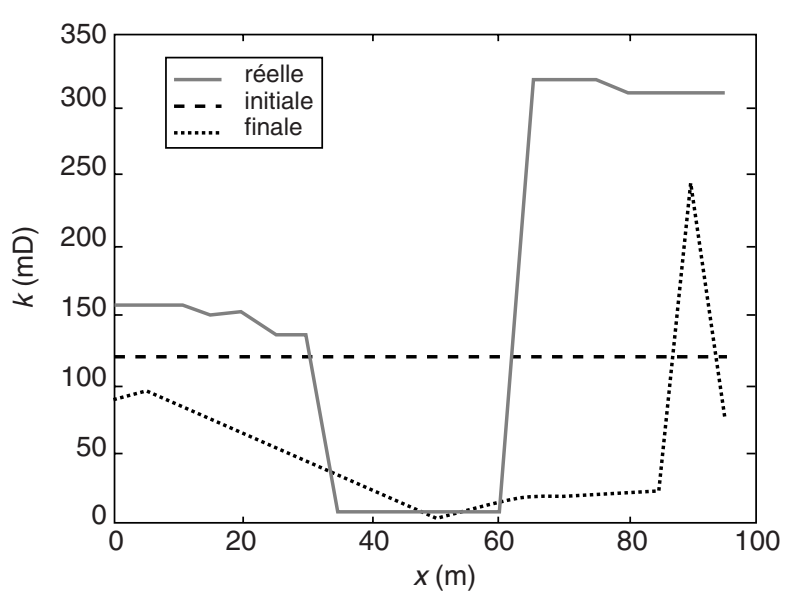

Figure 8a

Répartition des perméabilités.

Distribution of permeability.

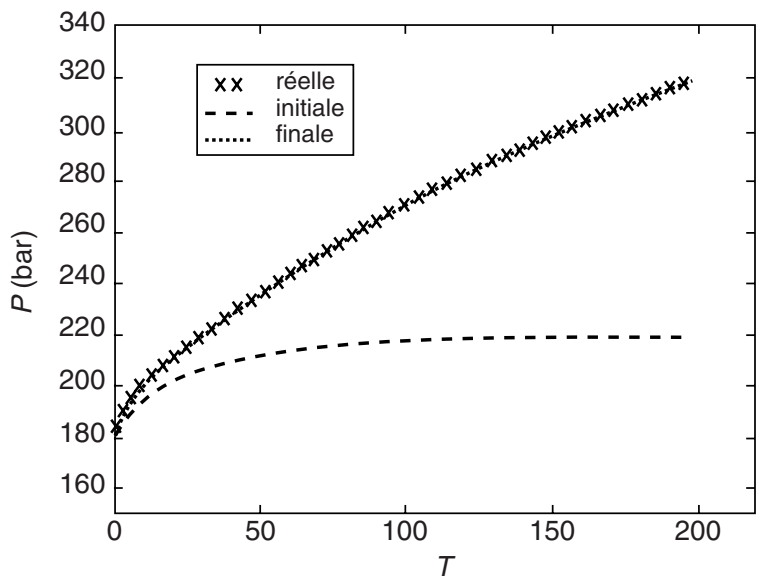

Figure $8 b$

Pression au puits injecteur.

Pressure at the injector well.

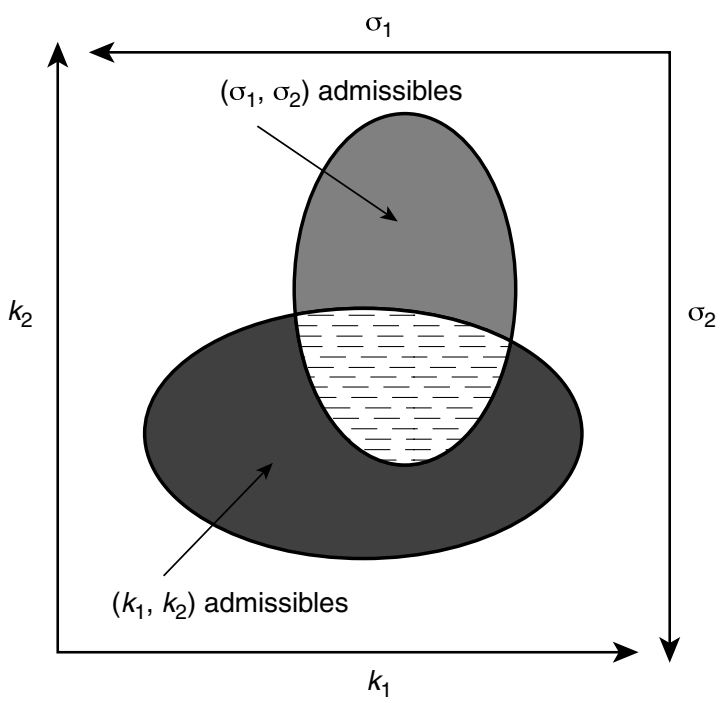

Figure 9

La solution du problème couplé est obligatoirement dans la réunion des solutions de chaque problème.

The solution of the coupled problem is in the intersection of the two domains.

\subsection{Relations perméabilité-impédance}

Dans cette première partie de thèse, on a décidé, dans un souci de simplicité, d'utiliser des relations de laboratoire, des relations algébriques, trouvées dans la littérature.

On peut relier la perméabilité et l'impédance en passant par la porosité.

\subsubsection{Relation perméabilité-porosité}

Le premier paramètre auquel on essaie de corréler la perméabilité $k$ est bien évidemment la porosité $\phi$. En réalité, s'il existe des exemples de très bonnes corrélations, cette relation est le plus souvent très floue. On peut, en général, expliquer facilement la relation $\phi-k$ en s'appuyant sur un modèle de géométrie poreuse tel que le modèle intergranulaire, pour ne citer que le plus utilisé.

L'espace intergranulaire présente une relation $\phi-k$ assez bien connue expérimentalement, au moins dans le cas de grains de forme subsphérique et de granulométrie constante (Jacquin, 1964, par exemple, dans Bourbie et al., 1986).

Si l'on prend un milieu poreux très simplifié formé de capillaires droits de rayon $R$ et de porosité $\phi$, la formule de Poiseuille donne :

$$
k=\frac{R^{2}}{8} \phi
$$

Pour un réseau de capillaires tortueux de section quelconque, on trouve une formule du même type en introduisant le rayon hydraulique $R_{h}$, la tortuosité des lignes de courant $\tau(\phi)$ (en pratique, $\tau(\phi)$ varie de 0,1 pour les argiles à 0,7 pour le sable) et un paramètre $A$, peu variable :

$$
k=\frac{A}{\tau(\phi)} R_{h}^{2} \phi
$$

Mais $R_{h}$ est fonction de $\phi \sqrt{S}, S$ étant la surface spécifique de l'espace poreux. Comme $\sqrt{S}$ est elle-même fonction inverse du diamètre du grain $d$, Carman et Kozeny ont établi 
pour la perméabilité d'un tel réseau la formule (Carman, 1961, dans Bourbie et al., 1986):

$$
k=B \phi^{3} \frac{d^{2}}{\tau(\phi)}
$$

où $B$ est une constante pour un milieu donné.

Le paramètre $k / d^{2}$ est une fonction de $\phi^{n}, n$ variant expérimentalement, suivant la porosité, de 7 ou plus pour des porosités faibles $(\phi<5 \%)$ à moins de 2 pour les milieux poreux non consolidés $(\phi>>30 \%)$. Dans la formule de CarmanKozeny, si la tortuosité est constante, l'exposant $n$ est égal à 3 et c'est bien celui qu'on observe dans le verre fritté ou le grès de Fontainebleau pour des porosités comprises entre 15 et $30 \%$. De nombreux milieux intergranulaires naturels ont des porosités comprises entre 10 et $25 \%$. On prend donc souvent pour ces milieux un exposant compris entre 4 et 5 .

\subsubsection{Relation impédance-porosité}

Les équations de Wyllie pour la diagraphie sonique $\Delta t$ et pour la diagraphie de densité $\phi$ peuvent être combinées pour exprimer l'impédance acoustique en fonction des paramètres pétrophysiques du milieu, notamment de la porosité $\phi$ (Boyer et Mari, 1994). En effet, nous avons, dans le cas de formations «propres » (i.e. sans argile):

$$
\begin{gathered}
\Delta t=\phi \Delta t_{f}+(1-\phi) \Delta t_{m a} \\
\varphi=\varphi \varphi_{f}+(1-\phi) \varphi_{m a}
\end{gathered}
$$

De plus, on a, pour l'impédance $\sigma$ :

$$
\sigma=\varphi V=\frac{\varphi}{\Delta t}
$$

En combinant les équations (11), (12) et (13), on obtient finalement :

$$
\phi=\frac{\varphi_{m a}-i_{p} \Delta t_{m a}}{i_{p}\left(\Delta t_{f}-\Delta t_{m a}\right)-\left(\varphi_{f}-\varphi_{m a}\right)}
$$

avec :

$\begin{array}{ll}\varphi & \text { porosité } \\ \sigma & \text { impédance acoustique } \\ \varphi_{f} \text { et } \Delta t_{f} & \text { densité et temps de transit de la formation } \\ \varphi_{m a} \text { et } \Delta t_{m a} & \text { densité et temps de transit de la nature }\end{array}$

Si l'on veut exprimer $\sigma$ en fonction de $\phi$, on obtient :

$$
\sigma=\frac{\varphi_{m a}+\phi\left(\varphi_{f}-\varphi_{m a}\right)}{\phi\left(\Delta t_{f}-\Delta t_{m a}\right)+\Delta t_{m a}}
$$

Pour une formation avec argile, la relation est plus compliquée, mais issue du même raisonnement. À partir des deux diagraphies :

$$
\begin{gathered}
\Delta t=\phi \Delta t_{f}+\left(1-\phi-V_{s h}\right) \Delta t_{m a}+V_{s h} \Delta t_{s h} \\
\varphi=\varphi \varphi_{f}+\left(1-\phi-V_{s h}\right) \varphi_{m a}+V_{s h} \varphi_{s h}
\end{gathered}
$$

on obtient :

$$
\phi=\frac{\left(1-V_{s h}\right)\left(\varphi_{m a}-\sigma \Delta t_{m a}\right)+V_{s h}\left(\varphi_{s h}-\sigma \Delta t_{s h}\right)}{\sigma\left(\Delta t_{f}-\Delta t_{m a}\right)-\left(\varphi_{f}-\varphi_{m a}\right)}
$$

avec :

$V_{s h} \quad$ pourcentage d'argile

$\varphi_{s h}$ et $\Delta t_{s h}$ densité et temps de transit de l'argile

\subsubsection{Relation perméabilité-impédance}

Nous pouvons donc obtenir une relation directe entre la perméabilité du milieu et son impédance acoustique en combinant les équations de Wyllie avec celles de Carman ; on arrive alors à la formulation suivante, pour une formation « propre » :

$$
k=B\left(\frac{\varphi_{m a}-\sigma \Delta t_{m a}}{\sigma\left(\Delta t_{f}-\Delta t_{m a}\right)-\left(\varphi_{f}-\varphi_{m a}\right)}\right)^{3} \frac{d^{2}}{\tau(\phi)}
$$

et pour une formation avec argile :

$$
k=B\left(\frac{\left(1-V_{s h}\right)\left(\varphi_{m a}-\sigma \Delta t_{m a}\right)+V_{s h}\left(\varphi_{s h}-\sigma \Delta t_{s h}\right.}{\sigma\left(\Delta t_{f}-\Delta t_{m a}\right)-\left(\varphi_{f}-\varphi_{m a}\right)} \frac{d^{2}}{\tau(\phi)}\right.
$$

Notons la relation $f$ : on a ainsi $k=f(\sigma)$.

\subsection{Problème couplé}

En reliant ainsi les deux paramètres $k$ et $\sigma$, on peut alors les inverser simultanément. On part d'un modèle initial, avec une impédance et une perméabilité dans chaque maille, liées par la relation $f$. On applique sur ce modèle le simulateur sismique et le simulateur d'écoulement, on obtient des réponses en pression (au puits injecteur) et en vibration (à la surface). On calcule alors la fonction coût de chaque problème en utilisant la relation $f$. De ce fait, à chaque pas $n+1$ des deux boucles d'inversion, nous réactualisons les deux paramètres ainsi :

$$
\begin{gathered}
k^{n+1}=k^{n}-\alpha_{k} \nabla \mathbf{J}_{p^{n}}\left(\mathrm{k}^{\mathrm{n}}, \sigma^{n}\right) \\
\text { où : } J_{p^{n}}\left(k^{n}, \sigma^{n}\right)=\mu_{k} \int_{0}^{T}\left(p^{n}(0, t)-\hat{p}(t)\right)^{2} \mathrm{~d} t+\tau_{k}\left\|k^{n}-f\left(\sigma^{n}\right)\right\|_{2}^{2} \\
\sigma^{n+1}=\sigma^{n}-\alpha_{\sigma} \nabla J_{y^{n}}\left(\sigma^{n}, k^{n}\right) \\
\text { où : } J_{\sigma^{n}}\left(\sigma^{n}, k^{n}\right)=\mu_{\sigma} \int_{0}^{T}\left(y^{n}(0, t)-\hat{y}(t)\right)^{2} \mathrm{~d} t+\tau_{\sigma}\left\|\sigma^{n}-f\left(k^{n}\right)\right\|_{2}^{2}
\end{gathered}
$$

$\mu_{k}, \tau_{k}, \mu_{\sigma}$ et $\tau_{\sigma}$ sont des poids à déterminer « manuellement » pour compenser les différences dans les échelles de valeurs.

$\alpha_{k}$ et $\alpha_{\sigma}$ sont les pas des inversions calculés par l'algorithme de Goldstein (voir 2.2).

Remarque : Nous n'utilisons pas ici d'informations a priori sur les distributions d'impédances ou de perméabilités. C'est un choix réfléchi: nous voulons prouver que sur un 


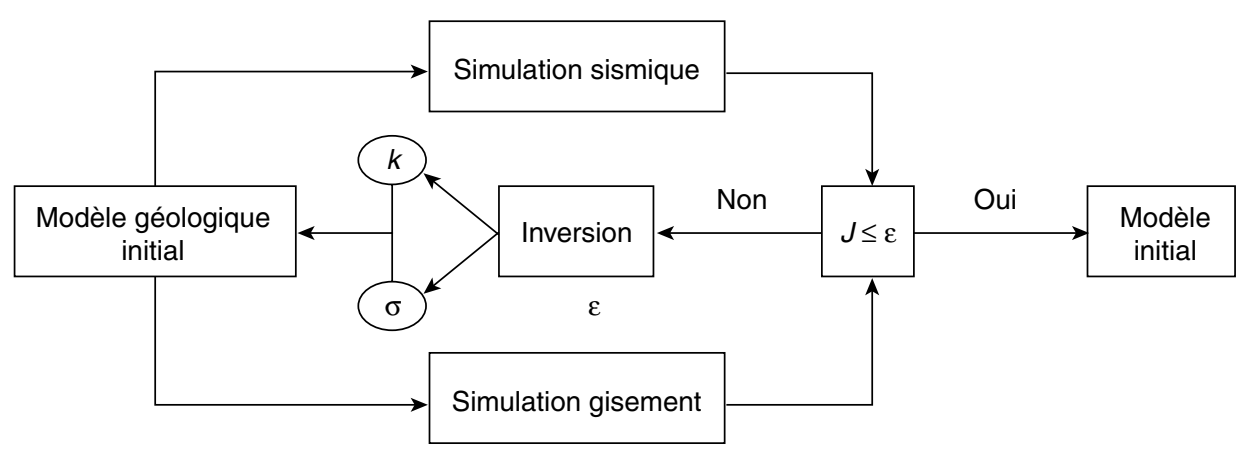

Figure 10

Algorithme de l'inversion couplée.

Coupled inversion loop.

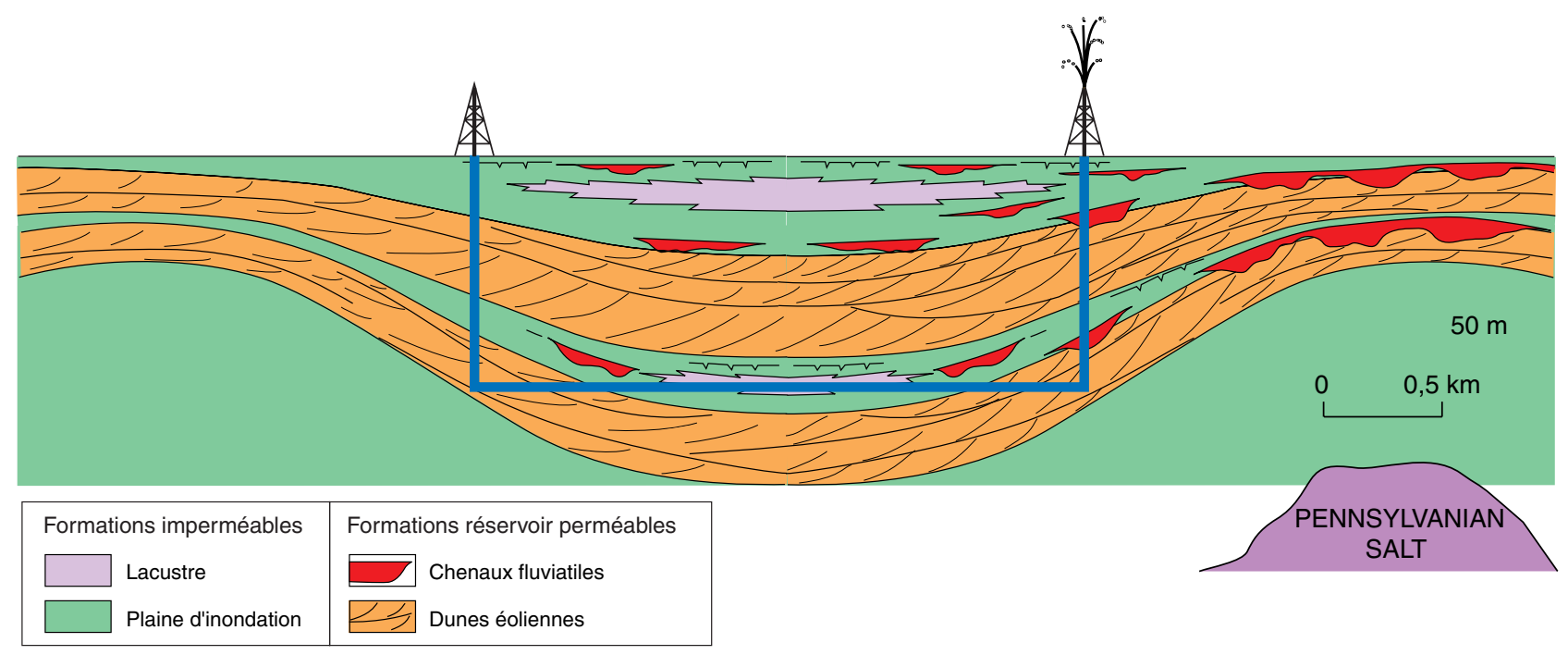

Figure 11

Exemple géologique d'un réservoir synclinal isolé par une couverture et une base imperméable éolien-permien de l'Utah. Geologic example of a Utah Eolian-Permian synclinal reservoir isolated by overlying and underlying impermeable beds.

cas simple, en partant d'une répartition très éloignée de la solution recherchée (exemple : répartition constante, voir 4.2), l'inversion couplée améliore grandement les résultats.

L'inversion se fait en utilisant, là aussi, la méthode de l'état adjoint pour le calcul du gradient (fig. 10).

\section{RÉSULTATS}

Voyons maintenant comment se comporte le code de calcul. Nous allons montrer que l'addition des informations sismiques à des tests d'écoulement améliore l'inversion des deux paramètres $k$ et $\sigma$. Nous avons, pour cela, construit un modèle synthétique qui peut prendre en compte la sismique et un écoulement. L'intérêt vient surtout de la capacité à retrouver, grâce au couplage, des informations perdues avec une inversion classique, comme de forts gradients dans la répartition des deux paramètres.

L'exemple géologique schématisé en figure 11 offre une configuration classique et parfaite pour illustrer nos résultats. Le réservoir synclinal isolé par une couverture et une base imperméable nous donne un fort gradient de perméabilité et d'impédance. Attention, nous nous sommes juste inspirés de la configuration physique ; il n'y avait pas de valeur des paramètres pétrophysiques associés au modèle. Cela n'a que peu d'importance pour prouver l'amélioration apportée par notre 


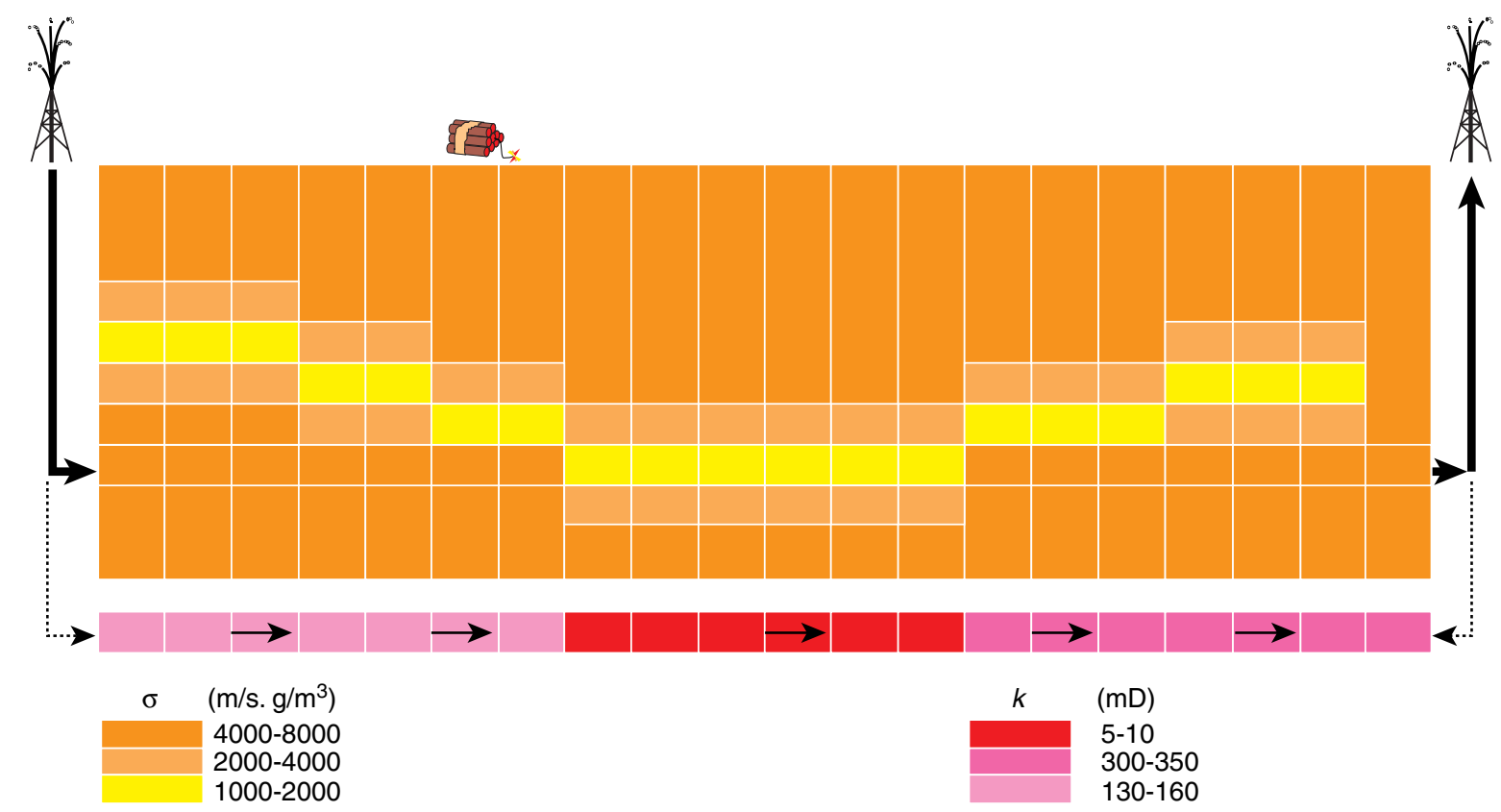

Figure 12

Schéma du modèle synthétique maillé.

Synthetic meshed model.

méthode par rapport aux inversions classiques, du moment que tous les tests sont faits sur les mêmes valeurs. On a donc informé le modèle avec des valeurs de perméabilité et d'impédance cohérentes avec les différents faciès présents sur l'exemple géologique (fig. 12).

On a ensuite modélisé un écoulement 1D qui traverse l'éolien et la plaine d'inondation. Puis, on a procédé à une étude multi-1D sismique au-dessus du bassin constitué des trois milieux : éolien, plaine d'inondation, éolien.

Il est important de noter que si l'apport d'information pour l'inversion en gisement est important (car on peut relier dans chaque maille de l'encaissant la perméabilité à l'impédance), l'importance en est moindre pour le côté sismique, puisqu'il n'y a qu'une perméabilité ajoutée dans chaque colonne sismique 1D étudiée. Nous verrons pourtant que l'amélioration apportée par le couplage se retrouve dans l'inversion de chaque paramètre.

\section{Résultats numériques}

Observons d'abord les résultats au niveau de l'inversion de la perméabilité dans l'encaissant sur les figures 13a et $13 \mathrm{~b}$.

On peut immédiatement constater que la répartition de perméabilité trouvée par la méthode couplée est beaucoup plus proche de la répartition recherchée (celle qui a servi à créer les données) que celle calculée par la méthode classique. La réponse en pression correspondante cale quant à elle parfaitement avec les données « observées » au puits injecteur.

En revanche, il faut signaler que doubler, voire tripler le nombre d'itérations n'apporte que peu d'améliorations à la répartition (fig. 14a). Cela est sans doute dû à la relation entre $k$ et $\sigma$, qui est prise en compte dans les calculs. Des tests seront faits sur ce sujet.

Néanmoins, le résultat essentiel est que le saut de perméabilité (sa position et son amplitude relative) est restitué dans la répartition trouvée par notre code, alors qu'il était énormément « lissé » par la méthode classique. Un tel résultat est à lui seul très encourageant. Mais nous pouvons aussi nous apercevoir que l'opération est plus rapide par notre méthode. En effet, la comparaison des deux fonctions coûts (fig. 14b) montre que, si celle de l'inversion couplée est très oscillante (un autre phénomène à étudier), elle reste très inférieure à partir d'une certaine valeur à celle de l'inversion classique, pratiquement à chaque pas d'itération. Attention, on a comparé ici les mêmes fonctions coûts, i.e. :

$$
J(k)=\frac{1}{2} \int_{0}^{T}(p(0, t)-\hat{p}(t))^{2} \mathrm{~d} t
$$

On améliore donc et la qualité du résultat, et la rapidité du temps de calcul.

Qu'en est-il maintenant de l'inversion du paramètre sismique ? Le peu d'information apporté suffit-il à améliorer le modèle? 


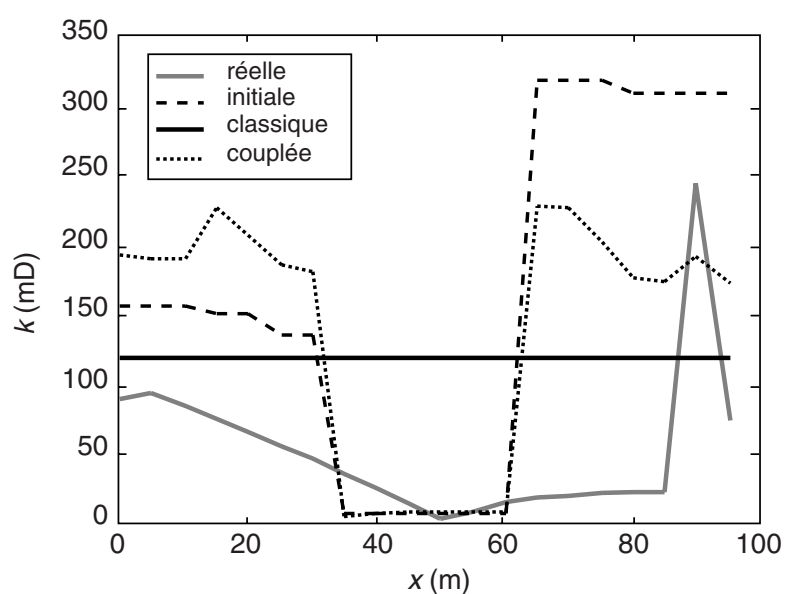

Figure 13a

Répartition des perméabilités.

Distribution of permeability.

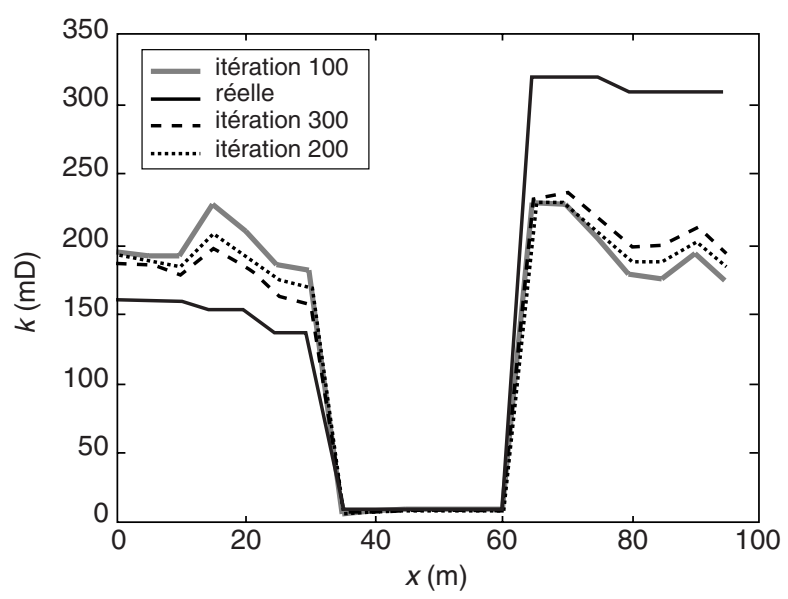

Figure 14a

Évolution des itérations.

Iterations.

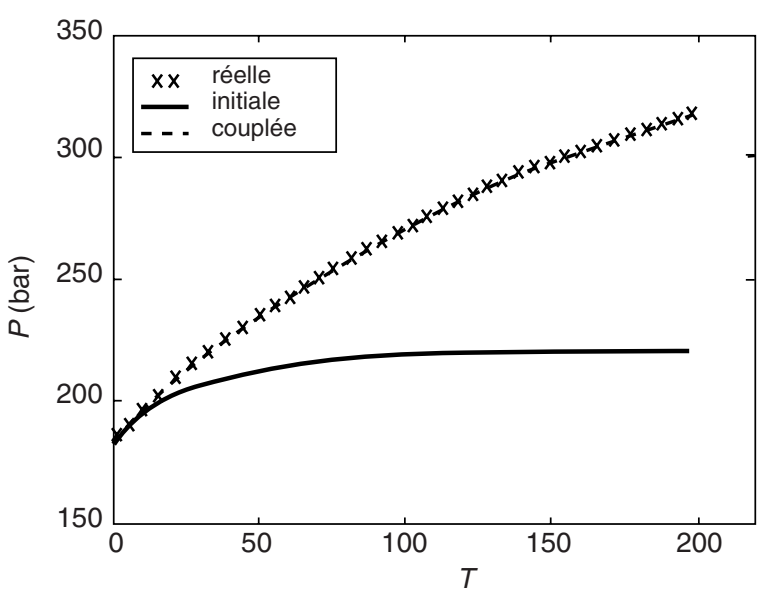

Figure 13b

Pression au puits injecteur.

Pressure at the injector well.

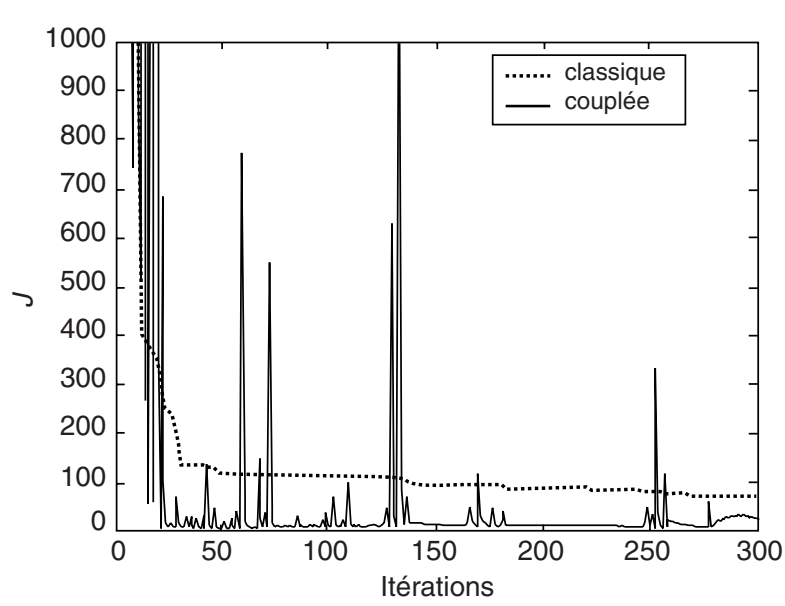

Figure 14b

Fonctions coûts.

Cost functions.
Le modèle maillé est composé de vingt colonnes. On en examinera deux.

Dans le cas présenté en figure 15a, l'inversion dite « classique » donnait déjà une bonne répartition des impédances acoustiques, et surtout, il y figurait déjà le fort gradient occasionné par le changement de faciès. Il faut remarquer néanmoins que le calcul couplé apporte une amélioration sensible au niveau de la valeur exacte des points, qui calent ici parfaitement avec les valeurs recherchées. En revanche, le progrès essentiel vient de la rapidité de l'algorithme, car la figure $15 \mathrm{c}$ montre que la fonction coût du calcul couplé décroît bien plus rapidement que celle du calcul classique. Encore une fois, on a comparé ici la même fonction, i.e. :

$$
J(\sigma)=\frac{1}{2} \int_{0}^{T}(y(0, t)-\hat{y}(t))^{2} \mathrm{~d} t
$$

Autrement dit, nous atteignons les écarts voulus entre les calculs et les observations plus rapidement grâce à notre méthode.

Voyons maintenant un deuxième cas, l'étude d'une deuxième colonne sismique.

Ici (fig. 16a, 16b, 16c), ce n'est pas la rapidité des calculs qui est améliorée (les fonctions coûts sont semblables, voir figure 16c), mais c'est la répartition des impédances qui est bien meilleure par la méthode couplée. Cette amélioration ne se situe pas au niveau du gradient d'impédance qui apparaissait déjà avec la méthode classique, mais plutôt au niveau des valeurs des impédances calculées (fig. 16a). L'écart, cette fois, avec les valeurs trouvées par l'inversion classique est important, et nettement à l'avantage de la méthode couplée. Nous trouvons une répartition des impédances très proche de la répartition recherchée, qui nous a servi à créer les données. 


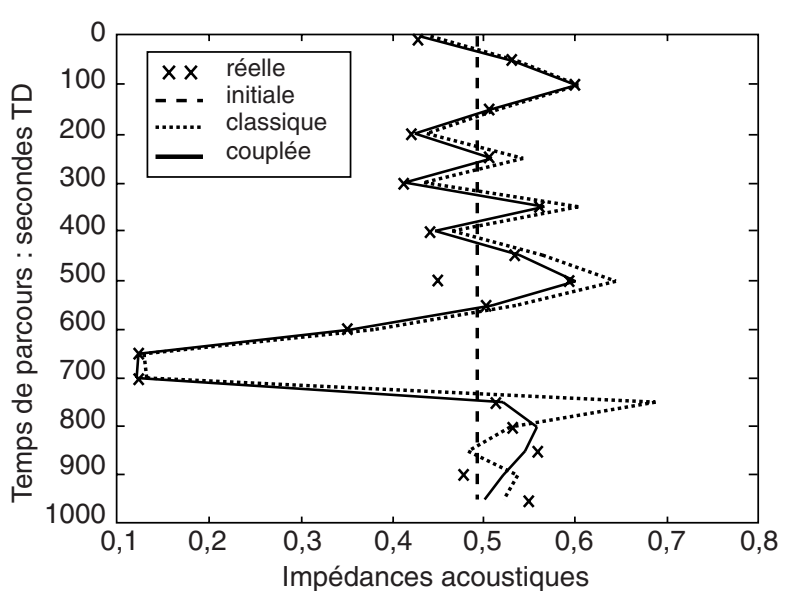

Figure 15a

Répartition des impédances.

Distribution of impedance.

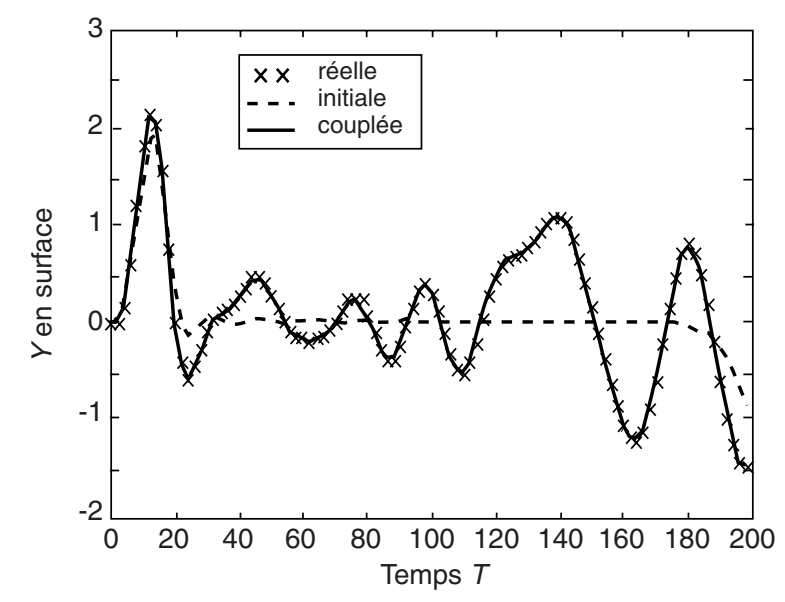

Figure 15b

Vibrations en surface.

Seismograms.

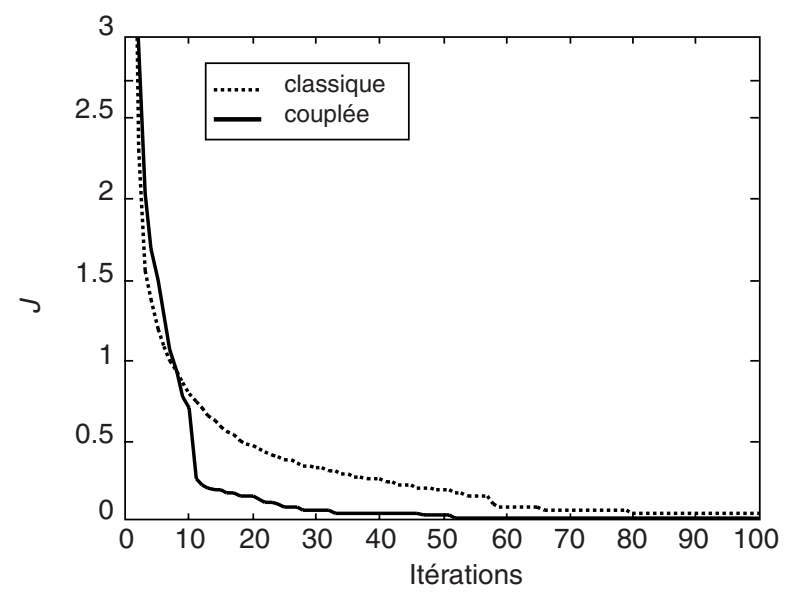

Figure 15c

Comparaison des fonctions coûts.

Comparison of cost functions.

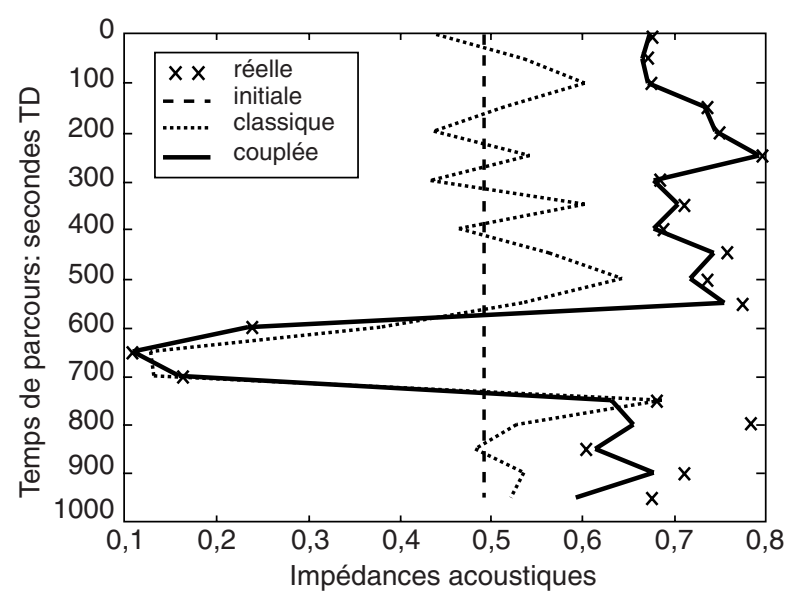

Figure 16a

Répartition des impédances.

Distribution of impedance.

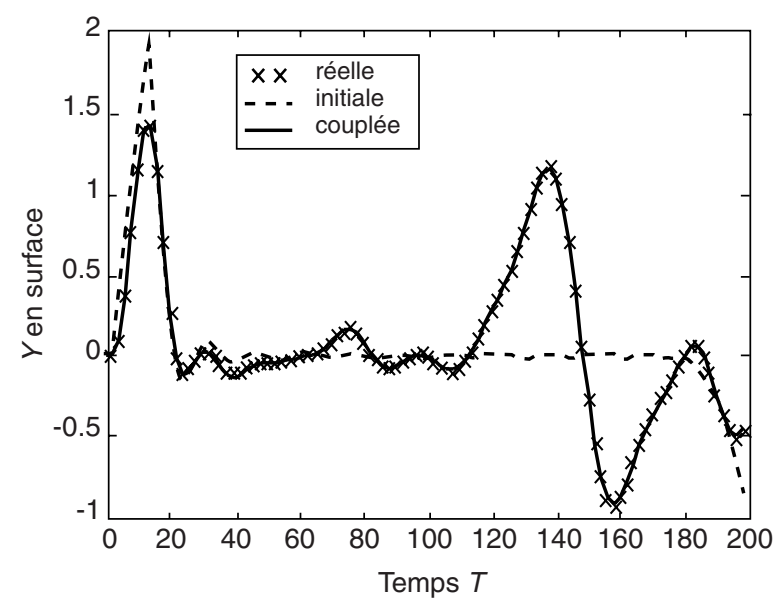

Figure 16b

Vibrations en surface.

Seismograms.

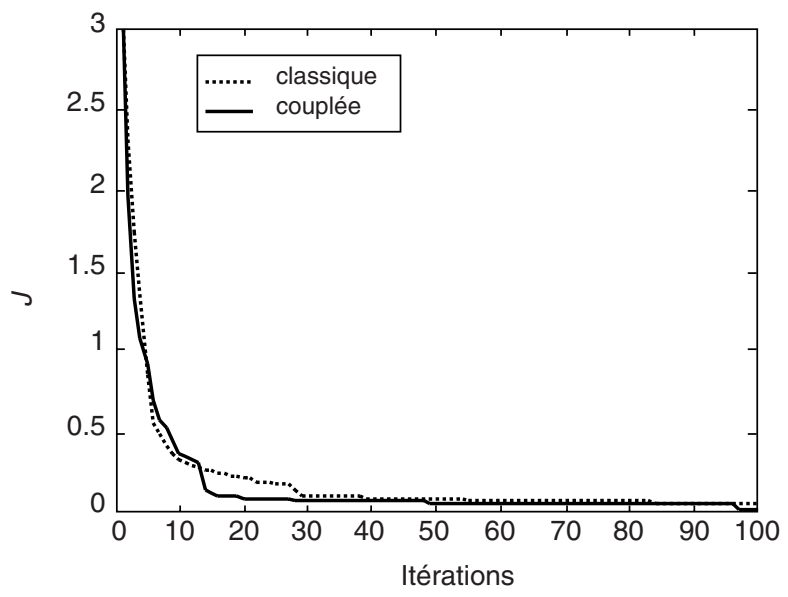

Figure 16c

Comparaison des fonctions coûts.

Comparison of cost functions. 
On constate donc sur ces deux exemples que l'amélioration de notre méthode couplée sur l'inversion sismique est significative, malgré le peu d'information ajouté. Le progrès se fait :

- soit au niveau de la rapidité des calculs, avec une fonction objectif qui décroît plus rapidement que par une inversion classique ;

- soit au niveau des valeurs des impédances retrouvées après inversion, valeurs plus proches de celles de la répartition recherchée.

\section{CONCLUSION}

Nous avons mis au point, durant cette première moitié de thèse, une méthode permettant de réaliser une inversion couplée des paramètres perméabilité absolue et impédance acoustique d'un modèle géologique. Elle est réalisée à partir de deux modèles directs classiques utilisés pour une simulation d'écoulement et une simulation d'acquisition sismique. Il s'agit de deux modèles 1D. L'inversion est faite grâce à des techniques couramment employées elles aussi, comme le calcul du gradient de la fonction coût par la méthode de l'état adjoint.

Le couplage est basé sur la relation entre les deux paramètres d'inversion. Nous sommes partis de relations de laboratoire, utilisées dans la littérature. Ce sont des relations qui traduisent la réalité physique seulement dans certaines configurations bien précises du sous-sol (relations de CarmanKozeny et équations de Wyllie). Mais ces relations algébriques présentent l'avantage d'être simples et facilement intégrables dans un code de calcul.

Nous avons réalisé un modèle synthétique, de configuration inspirée d'un cas géologique réel. Puis, une comparaison a été réalisée entre les méthodes d'inversion classiques employées en sismique et en gisement et notre méthode couplée.

Les premières conclusions montrent que le couplage permet de retrouver les forts gradients de perméabilité qui sont plus ou moins lissés par une méthode classique. Les plaines d'inondation et autres barrières imperméables sont donc localisées et reconstruites dans le modèle final. De plus, l'inversion se fait plus rapidement grâce au couplage, les calculs demandant moins d'itérations.

Pour l'inversion de l'impédance acoustique, même si elles sont moins flagrantes, les améliorations sont néanmoins significatives. Tout d'abord, les valeurs numériques des impédances calculées par l'inversion couplée sont plus proches des valeurs recherchées du modèle (celles qui servent à créer le modèle synthétique). Nous remarquons sur les graphiques que les répartitions retrouvées se superposent très bien avec les « réelles ». De plus, là aussi, les calculs se font dans l'ensemble, avec un nombre d'itérations moins important que par une inversion classique. Ce sera un élément déterminant lorsque la méthode sera appliquée à des cas réels, avec des simulateurs 3D professionnels.

\section{RÉFÉRENCES}

Bamberger, A., Chavent, G. et Lailly, P. (1977) Une application de la théorie du contrôle à un problème inverse de sismique. Les Annales de géophysique, 3.

Baranger, J. (1977) Introduction à l'analyse numérique, Collections Méthodes.

Bourbie, T., Coussy, O. et Zinzner, B. (1986) Acoustique des milieux poreux, Éditions Technip.

Bourdarot, G. (1990) Interprétation des essais de puits, Éditions Technip.

Boyer, S. et Mari, J.L. (1994) Sismique et diagraphies, Éditions Technip.

Céa, J. (1971) Optimisation : théories et algorithmes, Dunod.

Chavent, G. et Sabatier, P.C. (1996) Inverse Problems of Wave Propagation and Diffraction. Proceedings, Aix-les-Bains, France.

Dautray, R. et Lions, J.L. (1988) Analyse mathématique et calcul numérique pour les sciences et les techniques, Masson.

Daviau, F. (1986) Interprétation des essais de puits: les méthodes nouvelles, Éditions Technip.

Dequirez, P.Y. et Richard, V. (1990) Application of Inversion to Integrated Stratigraphic Interpretation. Revue de l'Institut français du pétrole, $\mathbf{4 5}, 3,397-416$.

Dercourt, J. et Paquet, J. (1997) Géologie. Objets et méthodes. Cours et exercices résolus, Dunod.

Edoa, P.F. (1999) Inversion de formes géologiques en ingénierie de gisements en utilisant les données de production. Thèse, Université Paul-Sabatier.

Faurre, P. (1988) Analyse numérique ; Notes d'optimisation, Ellipses.

Gallouët, T. (1992) An Introduction to Finite Volume Methods. Clamart.

Guérillot, D. et Roggero, F. (1995) Matching the Future for the Evaluation of Extreme Reservoir Development Scenarios. Proc. of Improved Oil Recovery, 8th European Symposium, Vienne, Autriche, 1, 229-240.

Guérillot, D., Lazaar, S. et Pianelo, L. (1998) Inverse Methods in Geoscience Modelling. A Review for Prospective. Proc. of ECMOR VI, Peebles, Écosse.

Jégou, S. (1997) Estimation des perméabilités relatives dans des expériences de déplacements triphasiques en milieu poreux. Thèse, Université Paris IX Dauphine.

Lailly, P. (1990) Blind Tests of Inversion Packages for Stratigraphic Interpretation. Revue de l'Institut français du pétrole, 45, 3, 301-326.

Lailly, P. et Mace, D. (1986) Solution of the VSP OneDimensional Inverse Problem. Geophysical Prospectings, 34, 1002-1021.

Lascaux, P. et Theodor, R. (1986) Analyse numérique matricielle appliquée à l'art de l'ingénieur. Tome 1, Masson.

Lavergne, M. (1986) Méthodes sismiques, Éditions Technip.

Mechler, P. (1982) Les Méthodes de la géophysique, Dunod.

Minoux, M. (1983) Programmation mathématique : théorie et algorithme. Tome 1, Dunod.

Pianelo, L. et Guérillot, D. (1999) Méthode pour former un modèle d'une formation géologique, contraint par des données dynamiques et statiques. Brevet d'invention, $\mathrm{n}^{\circ}$ d'enregistrement national 9911008. 
Pianelo, L. et Guérillot, D. (1999) Modélisation géologique contrainte par l'inversion simultanée des données dynamiques et sismiques : Étude 1D. Rapport interne IFP, $\mathrm{n}^{\circ} 45652$.

Rahon, D., Blanc, G. et Guérillot D. (1996) Hélios Reservoir Group. Gradient Method Constrained by Geological Bodies for History Matching. SPE 36568.

Raviart, P.A. et Thomas, J.M. (1992) Introduction à l'analyse numérique des équations aux dérivées partielles, Masson.
Serra, O. (1979) Diagraphies différées. Bases de l'interprétation. Tome 1 : Acquisition des données diagraphiques. Bull. Cent. Rech. Explor.-Prod.Elf-Aquitaine, Mémoire 1, Pau.

Tarantola, A. (1987) Inverse Problem Theory (Methods for Data Fitting and Model Parameter Estimation), Elsevier.

Manuscrit définitif reçu en février 2000 